\title{
Autonomous vans - the planning process of transport tasks
}

https://doi.org/10.1515/eng-2020-0006

Received Nov 01, 2019; accepted Dec 02, 2019

\begin{abstract}
Autonomous vehicles are gaining more and more followers and are becoming a reality. Although the total change of the transport system to an autonomous one still remains a big challenge, the development of navigation, control and supervision systems for vehicles gives hope for an autonomous future. The article describes the rules for choosing a route and the process of outsourcing transport tasks. The formulas for calculating the values of indicators characterizing the transport process are provided in the simple case when vehicles move along a fixed route where there are no disturbances. Additionally, the theory of Markov random systems was used to determine the system capacity, average queue length for servicing and the average number of requests.
\end{abstract}

Keywords: autonomous vehicle, route planning, transport tasks, Markov queue processes

\section{Introduction}

Progress in the construction of means of transport depends on the increased requirements for road safety, especially where there is high traffic. Road safety is a complex issue related to the development of technical and IT measures [1-3] logistics and management $[4,5]$ and legal regulations [6-8]. There are strong efforts on an EU

\footnotetext{
*Corresponding Author: Jacek Caban: University of Life Sciences in Lublin, Faculty of Production Engineering, Głęboka 28, 20-612 Lublin, Poland; Email: jacek.caban@up.lublin.pl Aleksander Nieoczym: Lublin University of Technology, Faculty of Mechanical Engineering, Department of Machine Design and Mechatronics, Nadbystrzycka 36, 20-618 Lublin, Poland; Email: a.nieoczym@pollub.pl

Agnieszka Dudziak: University of Life Sciences in Lublin, Faculty of Production Engineering, Głęboka 28, 20-612 Lublin, Poland; Email: agnieszka.dudziak@up.lublin.pl

Monika Stoma: University of Life Sciences in Lublin, Faculty of Production Engineering, Głęboka 28, 20-612 Lublin, Poland; Email: monika.stoma@up.lublin.pl
}

level towards harmonizing the European vision on connected and automated vehicles for the following decades like the Gear 2030 initiative that technical, legislation and financing challenges [9]. When designing a modern vehicle, particular attention is paid to ensuring a high level of safety for both vehicle travelers and other road users (other vehicles, pedestrians, etc.). It may be tested either by means of computer simulations or real crash experiments $[10,11]$. Initially the main motivation was to make driving easier or more comfortable, but world megatrends have oriented the development towards to lower fuel consumption, higher traffic safety and reduced environmental impact [9]. Research presented in [12-14] shows that still a human - the driver, of the vehicle and his reactions have an impact on road traffic safety.

Technical progress in the construction of autonomous vehicles (AV) is associated with the use of navigation systems that enable them to move in urban traffic. These systems are primarily GPS navigation, adaptive distance radar or lane tracking. Near Field Communication (NFC) also provides great potential in transport services [15]. Despite many years of research into the autonomy of vehicles, there are still various problems to be solved. On the technical side the lack of precise solutions for locating the vehicle position is a problem [7]. New solutions are sought, based e.g. on coupling a GPS unit with an inertial unit (Inertial Measurement Union). Sensors are used that go beyond human senses, and some of the sensors can only work reliably in only good weather conditions. One of the most important legal obstacle is that vehicles without mentally and physically capable drivers are generally not permitted in public traffic [7].

The use of AV vehicles for commercial tasks related to the distribution of goods or the role of a taxi requires additional analysis. These include: calculation of driving time, route selection, the possibility of changing the route to an alternative one, the order of handling points, the number of waiting points for service or waiting time. The mentioned indicators characterizing the service process are based on probability and mathematical statistics [16]. In addition, elements of service systems and queuing system analysis are used.

○ Open Access. (C) 2020 A. Nieoczym et al., published by De Gruyter. (cc) BY 4.0 License 


\section{Services market with the use of autonomous vehicles}

Autonomous vehicles that act as taxis, passenger cars or trucks are already being tested on the roads and legal regulations are prepared to allow such vehicles to be used. The problem of allowing autonomous vehicles to move on public roads is described in [17]. The assumptions of the vehicle stream model are described, which makes it possible to infer the type of interaction between human-guided vehicles and $\mathrm{AV}$ vehicles. The described algorithm allows theoretically and numerically to study the conditions of linear stability of a heterogeneous vehicle stream, which takes into account probabilistic speed changes, and the stochastic order of vehicle types in the stream. Creating mathematical models of autonomous vehicle movement is based on modification and testing of hypotheses formulated on the basis of data collected from human-driven vehicles. New navigation tools are also being created that sometimes replace existing solutions. An example is emerging new high-resolution maps for autonomous cars created by the corporation Toyota Research Institute-Advanced Development (TRI-AD) and Carmera. These maps are to be the basis of the open Automated Mapping Platform (AMP) [18]. The construction of programs supervising the sending of autonomous vehicles to perform tasks is implemented based on the collection of data from urban traffic of passenger cars and delivery vans, acceptance and execution of transport orders [18-20]. A good example is the taxi traffic coordination system, which forecasts the demand for taxi services, using historical data on services rendered, big data and information on the current situation in the city. Every half hour, the system forecasts the number of taxis needed in a $500 \mathrm{~m}^{2}$ area. For this, it uses artificial intelligence mechanisms with learning functions, based on taxi service registers, the availability of public transport, weather information and important events in the city. The program used allows to shorten the customer waiting time for a taxi, while increasing the fleet utilization rate. The system showed an efficiency of $94.1 \%$ [18].

According to analysts of the TSL industry, the greatest demand will be for vans moving in the city. This is due to the fact that according to the UN forecast, in $203078 \% \mathrm{Eu}$ ropeans and $70 \%$ Chinese people will live in cities. The city supply market is expected to be worth USD 86 billion a year. The final stage of delivering the parcel to the target recipient - between the sorting house and his apartment - will be $35 \div 50 \%$ shipping cost in general [21]. In some cases they are higher and reach up to $60 \%$ shipping cost in general. In addition, online sales and related mail-order sales will be more widespread. Shipping subscriptions already (e.g. in auction sites) have already resulted in a quantitative and valuable increase in sales.

Below are two conceptual autonomous delivery vehicles. Renault EZO-Pro vehicle with dimensions $5 \times 2 \times 2$ meters (length $\mathrm{x}$ width $\mathrm{x}$ height), the chassis is designed to fit as large a cabin as possible. The wheels have been extended to the corners and protected so that they do not pose a threat to pedestrians. The engine is powered by batteries located flat under the floor, which allow travel of about $400 \mathrm{~km}$ [21].

Another vehicle - EV Nuro is to be a mobile parcel locker. The car weighs about $680 \mathrm{~kg}$, of which the payload is about $115 \mathrm{~kg}$ and moves at a speed of $30 \mathrm{~km} / \mathrm{h}$. The length and height correspond to the dimensions of the van, but it is very narrow - the width is about 1 meter. The vehicle is able to bypass the child without changing the occupied lane, which reduces the risk of collision with other road users [18]. In 2017, Ford began testing autonomous pizza delivery, Amazon and Pizza Hut intend to provide similar services using Toyota's e-Palette (Toyota's electric de-

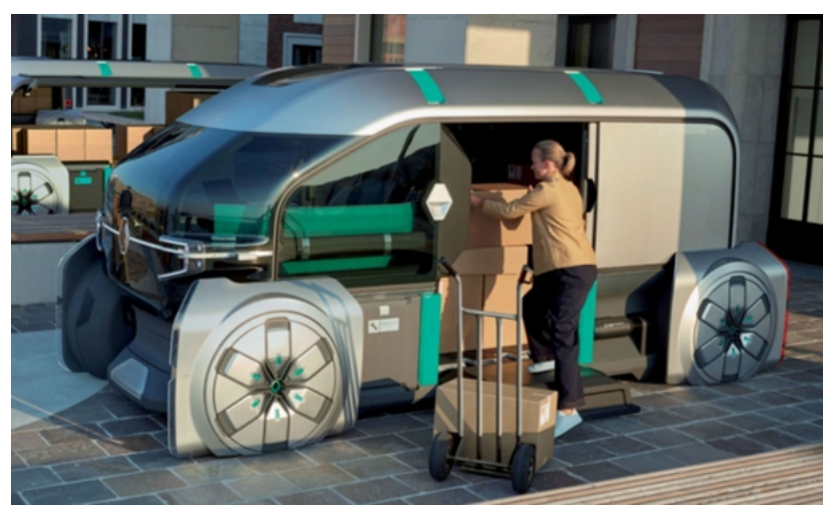

Figure 1: EZO-Pro autonomous delivery vehicle [21].

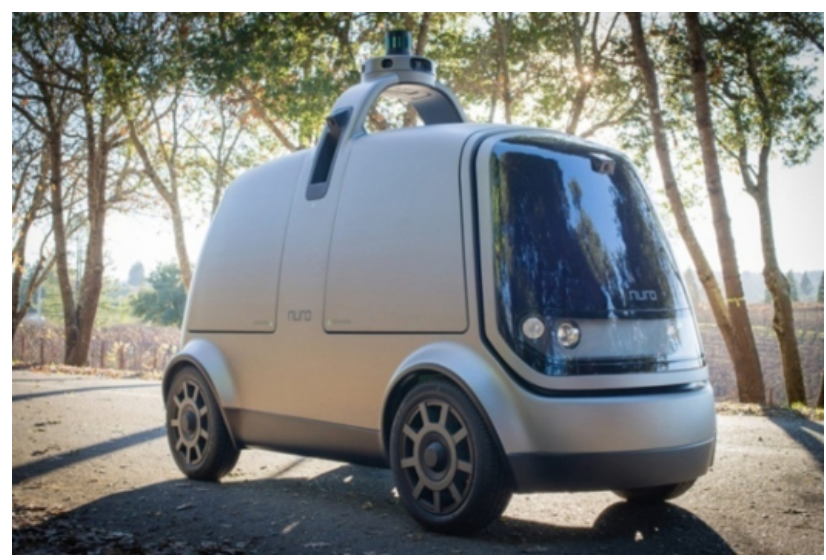

Figure 2: Nuro autonomous vehicle [18]. 
livery vehicles (Uber, Mazda and DiDi also participate in the project).

\section{Modeling autonomous vehicle in traffic conditions}

The condition for admission of autonomous vehicles to traffic is their ability to react dynamically to hazards and road situations. Another issue is the creation of a methodology for determining the route for defined posting and collection points.

The issue of using autonomous vehicles to operate the parcel collection station is described in [22]. The goods must be delivered from the warehouse to the preferred pickup station or another station in the immediate area. Autonomous vehicles move between the depot and stations. Decision support where and when to send the vehicle was based on the intuitive PFA (Please Find Attached) algorithm. PFA enables real-time decision making, balancing the trade-off between fast delivery and delivery consolidation. It has been proposed to combine PFA with an approximation of the value function to take into account the heterogeneity of demand. Modeling results provide input for strategic and tactical planning of cargo deliveries in the urban area.

Modeling the $\mathrm{AV}$ vehicle behavior when reaching the reporting point and searching for a place to operate this point is described in [23]. For modeling, the results of observing identical behaviors in the case of humanguided vehicles were used. Analyzes were performed using structural least-squares (PLS-SEM) structural equations, partial-least-squares (PLS-MGA) analysis to develop observed heterogeneity and partial least squares orientation (PLS-POS) to study unobserved heterogeneity. The problem of calculating the $\mathrm{AV}$ vehicle travel time to the destination is also described in [24]. Two simulations were carried out: the first based on historical traffic data, the second based on real-time traffic information. They were used to test the reliability of route search and to make route length probability distributions depending on driving time. The tool to support the management of the fleet of individual public autonomous vehicles (VIPA) can be the VIPAFLEET program [25]. The option of treating VIPA as vehicles delivering goods or taxi vehicles is based on the theory of flows in the extended time network. This allows you to calculate the optimal offline solution, propose strategies for the online situation, and evaluate performance against the optimal offline solution. Route planning is an issue for which multi-channel service systems and analytical methods of queuing system analysis are used.

\section{Planning the route of the vehicle}

Autonomous vehicles together with send and receive points form single-channel or multi-channel service systems. Means of transport can move between delivery / collection points along fixed routes or freely. The free route selection is dynamic when the vehicle is moving. Dynamic route selection has been the subject of research using the Artificial Immune System (AIS). The system is configured in such a way that, in response to changes, it adapts dynamically to the environment and in real time, and sends new tasks to AV vehicles [26, 27].

Planning the optimal route of AV vehicles based on differential equations is described in [28]. Route planning includes: adopting the probability distribution of flows, calculating the distribution of time-optimal paths for the above flow distribution together with solving the stochastic dynamic flow equations. The last stage is the calculation of the risk of uncertainty about the anticipated driving times and the route. The presented mathematical model has been verified for data of idealized canonical flow scenarios commonly found in urban environments.

All time and route planning issues can be classified as [29]:

- general route calculation,

- routeoptimization,

- setting a custom route, divided into:

- static methods in which the route previously allocated remains unchanged until the vehicle completes the route,

- methods of time windows in which the route segment can be used by different vehicles in different windows of time,

- dynamic methods in which the use of any route segment is dynamically allocated to a moving vehicle.

In the process of designing autonomous systems, many decision variables are taken into account [30]:

- topography of transport routes,

- vehicle traffic management: anticipating and avoiding collisions and congestion,

- the number and arrangement of delivery / collection points,

- demand for transport vehicles, 
- issuing instructions for transport vehicles,

- routing transport vehicles,

- locatingfreevehicles,

- controlling the process of charging storage batteries of transport vehicles,

- handlingdamaged transport vehicles.

The concept of the arrangement of transport routes contains information on both permanently located transit routes and the vehicles themselves that travel along these routes in order to reach the appropriate loading / unloading places. Traffic management is required when it is possible that several vehicles may be in the same place at the same time, which leads to collisions. Based on information about the planned route, you can make decisions regarding the order of service for individual delivery / pickup points. After completing the transport task, the autonomous vehicle should go to the designated place and wait there for the assignment of the next task.

\section{The process of ordering transport tasks}

The process of sending autonomous vehicles for transport tasks should be considered through the rules for selecting the appropriate vehicle to perform a specific transport task [31, 32]. This problem occurs when there is a need to transport people or goods from a specific location to the destination. It can be solved in two ways: in the first case, the load can be assigned to a free vehicle. In the second case, a means of transport that is free at a given moment can be directed to a specific delivery / pickup point that requires transport at a given moment. The problem of separating transport tasks can be divided into two categories:

- tasks initiated by delivery / collection points (search for free vehicles),

- tasks initiated by vehicles (search for freeloads).

In static (off-line) control systems, all information about transport tasks is available when the transport process begins [33]. As a result, vehicles can be assigned to loads optimally, by formulating the shipping problem as an assignment problem. In the case of dynamic (online) control systems, simple heuristics operating according to the FIFO (first-come-first-served) rule are used to solve the transport task. The first free vehicle is sent to the load that first requested transport service. The control process can also be carried out according to the FEFS (first-encountered-first-served) rule. This rule is used in distributed dynamic control systems. Transport vehicles, capable of transporting multiple loads at the same time, take the first free loads encountered if they have space for them. Then the transport modules are unloaded at their destination. Simulation studies have shown that for singleloop cases of this type of FEFS heuristics are more effective than those that use the FIFO rule. A set of heuristic rules has been defined that can be used in distributed control systems for shipments initiated by delivery / collection points:

- any vehicle rule: the transport task is randomly assigned to a free vehicle, regardless of its position relative to the load,

- nearest vehicle rule: the transport task is assigned to the nearest slow vehicle,

- the furthest vehicle rule: a transport task is assigned to a vehicle that is furthest from the load that needs to be picked up,

- the longest waiting rule: a transport task is assigned to a vehicle that has the longest stopping time among all vehicles currently not having assigned transport tasks,

- least vehicle use rule: a transport task is assigned to a vehicle with a minimum average use (e.g. the ratio of hours worked to the sum of hours worked and parking).

The application of the last two rules is intended to balance the load on vehicles. Rules that include ship-initiated shipments (free load search) are defined as follows:

- any load rule: the vehicle is sent to a delivery / pickup point, selected randomly from all such points that require transport service at a given time,

- shortest travel or distance rule: the vehicle is sent to the nearest delivery / pickup point. The purpose of this rule is to minimize empty travel times for vehicles,

- longest queue rule: the vehicle is sent to the delivery / pickup point with the largest number of loads awaiting transport.

The issue of dynamic route planning can be divided into two types:

- total route planning,

- incremental route planning.

In total planning, in one approach, the entire route of the means of transport is planned from the starting point to the destination. Incremental planning consists in successive determination of successive sections of the route (e.g. segment by segment). The process continues until 
the vehicle reaches its destination. The disadvantage of total planning is the possibility of outdating the route during the transport task due to the occurrence of unforeseen events. In addition to determining a collision-free route, other important issues to consider are disruptions and interruptions in the operation of the transport system. The reason for the disturbance may be e.g. a vehicle breakdown, obstacle on the route or manual intervention of the system controller. The result of the disturbance may be the blockade of the transport vehicle and, as a result, the inability to complete the transport task that has been started earlier. Simulation methods are used to solve problems related to changing a previously planned route.

\section{Probabilistic evaluation of the operation of simple system}

Below is the method for calculating the values of indicators characterizing the transport process in the basic case when the vehicles are traveling on a fixed route without disturbances. An additional assumption is the reliability of vehicles and service points.

Let's assume that there are $N$ points requiring service by $m$ vehicles. Each point sends reports about the intensity $\lambda$ while the service intensity of the vehicle is marked by $\mu$. The general intensity of notifications depends on the task load of a given point, i.e. it is a function of the system state. Assuming, furthermore, that the sequence of intervals between consecutive moments of sending a notification by service points is recursive, i.e. these intervals are independent of each other and have the same distribution. The service time is a random variable and with $m$ vehicles each of them works independently of each other and the service time has the same distribution. In order to determine the system capacity, the average queue length to handle and the average number of requests, the theory of Mark's random systems can be used [16]. It is assumed that it is a single-channel system for which the $\lambda$ notification stream is described by the Poisson distribution while the service time is subject to the exponential distribution. Operation is carried out according to the FIFO rule. The authors have identified the following system states are possible:

$\mathrm{E}_{0}$ - all vehicles free, no tasks to handle,

$\mathrm{E}_{1}$ - one vehicle busy, one notification in the system,

$E_{2}$ - two vehicles occupied, two notification in the system, :

$\mathrm{E}_{m}-m$ vehicles used, $\mathrm{m}$ notifications in the system,
$\mathrm{E}_{j}-m$ of vehicles busy, $j-m$ notifications in the queue for service,

:

$\mathrm{E}_{N}-\mathrm{m}$ vehicles occupied, $\mathrm{N}$ points awaiting service, $\mathrm{N}-\mathrm{m}$ requests pending service.

Between the individual states from $E_{1}$ to $E_{2}$ the system is carried out through a stream of notifications with an intensity $(N-1) \lambda$ because in state $E_{1}$ one service point has already sent a notification, so the possibility of notifications already has $(N-1)$ points. Between states $E_{0}$ to $E_{N}$, the system is carried out through a stream of notifications with an intensity of $N \lambda$. Using the mnemotechnical rule, you can write a system of differential equations describing the dynamics of the system (see Eq. 1):

$$
\begin{aligned}
p_{0}^{\prime}(t) & =-N \lambda p_{0}(t)+\mu p_{1}(t) \\
p_{1}^{\prime}(t) & =N \lambda p_{0}(t)-[(N-1) \lambda+\mu] p_{1}(t)+2 \mu p_{2}(t) \\
p_{i}^{\prime}(t) & =(N-i+1) \lambda p_{i-1}(t)-[(N-i) \lambda+i \mu] p_{i}(t) \\
& +(i+1) \mu p_{i+1}(t) \\
& \text { for } \quad 1 \leq i \leq m-1 \\
p_{m}^{\prime}(t) & =(N-m+1) \lambda p_{m-1}(t)-[(N-m) \lambda+m \mu] p_{m}(t) \\
& +m \mu p_{m+1}(t) \\
p_{j}^{\prime}(t) & =(N-j+1) \lambda p_{j-1}(t)-[(N-j) \lambda+m \mu] p_{j}(t) \\
& +m \mu p_{j+1}(t) \\
& \text { for } \quad m \leq j \leq N-1 \\
p_{N}^{\prime}(t) & =\lambda p_{N-1}(t)-m \mu p_{N}(t)
\end{aligned}
$$

Normalizing condition:

$$
\sum_{s=0}^{N} p_{s}(t)=1
$$

In the steady state we get the equations (see Eq. 3):

$$
\begin{aligned}
& 0=-N \lambda p_{0}+\mu p_{1} \\
& 0=N \lambda p_{0}-[(N-1) \lambda+\mu] p_{1}+2 \mu p_{2} \\
& 0=(N-i+1) \lambda p_{i-1}-[(N-i) \lambda+i \mu] p_{i}+(i+1) \mu p_{i+1} \\
& \text { for } \quad 1 \leq i \leq m-1 \\
& 0=(N-m+1) \lambda p_{m-1}-[(N-m) \lambda+m \mu] p_{m}+m \mu p_{m+1} \\
& 0=(N-j+1) \lambda p_{j-1}-[(N-j) \lambda+m \mu] p_{j}+m \mu p_{j+1} \\
& \text { for } \quad m \leq j \leq N-1 \\
& 0=\lambda p_{N-1} 0-m \mu p_{N} 0
\end{aligned}
$$

Steady state probabilities of the system can be written using the formulas:

$$
p_{i}=\frac{N !}{i !(N-i) !} \rho^{i} p_{0} \quad \text { for } \quad 1 \leq i \leq m
$$




$$
p_{j}=\frac{N !}{m ! m^{j-m}(N-j) !} \rho^{j} p_{0} \quad \text { for } \quad m+1 \leq j \leq N
$$

where: $\rho=\frac{\lambda}{\mu}$

The probability $p_{0}$ after is calculated from the dependence:

$$
p_{0}=\left[\sum_{i=0}^{m} \frac{N !}{i !(N-1) !} \rho^{i}+\sum_{j=m+1}^{N} \frac{N !}{m !(N-j) ! m^{j-m}} \rho^{j}\right]^{-1}
$$

The probability that $r$ points report the need for service:

$$
\begin{aligned}
& p_{m+r} \\
& =\frac{N ! \rho^{m+r}}{m !(N-m-r) ! m^{r}\left[\sum_{i=0}^{m} \frac{N !}{i !(N-i) !} \rho^{i}+\sum_{r=0}^{N-m} \frac{N ! \rho^{m+r}}{m^{r} m !(N-m-r) !}\right.}
\end{aligned}
$$

Average number of requests waiting in queue:

$$
\bar{v}=\frac{N !}{m !} p_{0} \sum_{r=0}^{N-m} \frac{r}{m^{r}(N-m-r)} \rho^{m+r}
$$

Average number of vehicles servicing points:

$$
\bar{m}=\sum_{i=0}^{m-1} i p_{i}+m\left(1-\sum_{i=0}^{m-1} p_{i}\right)
$$

Average number of requests in the system:

$$
\bar{n}=\sum_{i=0}^{m} i p_{i}+\sum_{j=m+r}^{N} j p_{j}
$$

Average time of notifications being in the system:

$$
t_{s}=\frac{\bar{n}}{\lambda(N-\bar{n})}
$$

Average time spent in the queue:

$$
t_{f}=t_{s}-\frac{1}{\mu}
$$

The above formulas allow for a preliminary calculation of the values of transport system indicators. They form the basis for detailed calculations. Operational research methods are used to analyze many transport tasks, e.g. in the work of municipal services in the city [34], postal services [35, 36], and modeling of public transport $[37,38]$.

\section{Conclusion}

Robotic equipment and autonomous driving systems have been approaching our surroundings and in the end, they will substitute humans step by step [39]. AGV (Automated Guide Vehicle) autonomous vehicles have found application in the industry for transporting supplies between workstations. The unchanging position of the served stations, constant distances between them and possible to calculate service times resulted in a mathematical apparatus based on probabilistic methods enabling the selection of the route and the order of service of the stations. The use of mathematical models related to the functioning of AVG vehicles for vehicles moving in urban traffic is not possible. The presence of other traffic participants, changing road conditions, the possibility of resignation from service causes and, in particular, stochastically appearing phenomena on the road cause the need to develop a completely different mathematical model of vehicle functioning, the need to create different traffic models and tasks performed by AV (Autonomous Vehicle) vehicles. The problem of choosing the route of an autonomous vehicle in urban traffic is a multi-criteria problem, the solution of which is possible using probabilistic methods in combination with queue theory. The methodology of calculating the values of indicators characterizing the transport process presented in the article is an approximate method that can be used in the initial design: route, time spent by an autonomous vehicle, waiting time for service and service time.

Mathematical models found in the literature describe only separate aspects of the autonomous vehicle movement, e.g. dynamic route change conditions, algorithms for changing the time and service point depending on changing road conditions. These models are still subject to verification using simulation tests collected from urban traffic data. So far, no attempt has been presented to link the published mathematical model of autonomous vehicle traffic into one unified methodology of functioning. For this reason, the authors of this article have presented equations that allow the calculation of indicators describing the service process. The presented equations give the opportunity to verify the mathematical model by simulation methods, e.g. using the Simulink program. Adding further model restrictions and checking the correctness of model operation can lead to the creation of equations describing the movement of an autonomous vehicle related to the service of points reporting such necessity. Next, the mathematical analysis must be supported by data describing the situation on the route sent in real time by a moving vehicle. The introduction of software enabling learning and route correction and quantities describing the service process will ensure obtaining real data describing the autonomous vehicle movement. 


\section{References}

[1] Droppa P. Application of thermal protection of military vehicles. ICMT'09: International Conference on Military Technologies. Edited by: Stefek, A., Jalovecky, R. 2010;47-54.

[2] Skrucany T, Kendra M, Stopka O, Milojević S, Figlus T, Csiszar C. Impact of the electric mobility implementation on the Greenhouse Gases production in Central European countries. Sustainability. 2019;11(18):4948. DOI: https://doi.org/10.3390/ su11184948

[3] Yu W, Egbelu PJ. Design of variable path tandem layout for automated guided vehicle systems. J. Manuf. Syst. 2001;20(5):305-19. DOI: https://doi.org/10.1016/S0278-6125(01)80050-9

[4] Gnap J, Poliak M, Semanova S. The issue of a transport mode choice from the perspective of enterprise logistics. Open Eng. 2019;9(1):374-83. DOI: https://doi.org/10.1515/eng-2019-0044

[5] Kampf R, Hlatka M, Bartuska L. Optimization of production logistics. Advances In Science And Technology-Research Journal. 2018;12(4):151-6. DOI: 10.12913/22998624/100351

[6] Poliak M, Mrnikova M, Simurkova P, Medvid P, Poliakova A, Hernandez $S$. Social law in road transport like tool safety road transport. XI International Science-Technical Conference Automotive Safety 2018, IEEE 2018, DOI: 10.1109/AUTOSAFE.2018.8373317

[7] Törő O, Bécsi T, Aradi S. Design of lane keeping algorithm of autonomous vehicle. Periodica Polytechnica Transportation Engineering. 2016;44(1):60-68, DOI: 10.3311/PPtr.8177

[8] Matijosius J, Vasiliauskas A, Vasiliene-Vasiliauskiene V. Krasodomskis $\check{Z}$. The assessment of importance of the factors that predetermine the quality of a service of transportation by road vehicles. 9th International Scientific Conference on Transbaltica, Vilnius, Lithuania, 07-08.05.2015. Proceedings of the 9th International Scientific Conference (Transbaltica 2015), Procedia Eng. 2016;134:422-9. DOI: https://doi.org/10.1016/j.proeng.2016.01. 034

[9] Szalay Z, Nyerges A, Hamar Z, Hesz M. Technical specification methodology for an automotive proving ground dedicated to connected and automated vehicles. Periodica Polytechnica Transportation Engineering. 2017;45(3):168-74. DOI: https://doi.org/ 10.3311/PPtr.10708

[10] Papis M, Jastrzębski D, Kopyt A, Matyjewski M, Mirosław M. Driver reliability and behavior study based on a car simulator station tests in ACC system scenarios. Eksploatacja i Niezawodnosc - Maintenance and Reliability. 2019;21(3):511-21. DOI: http://dx.doi.org/10.17531/ein.2019.3.18.

[11] Lubikowski K, Radkowski S. The project of shoulder of the suspension system for autonomous platform. The Archives of Automotive Engineering - Archiwum Motoryzacji. 2017;75(1):85-92. DOI: 10.14669/AM.VOL75.ART5;

[12] Matuszak Z, Jaśkiewicz M, Więckowski D, Stokłosa J. Remarks to the reliability assessment and to human actions - especially car driver. 18th International Scientific Conference-LOGI 2017, Ceske Budejovice, Czech Republic. MATEC Web of Conferences. 2017;134:00036. DOI: 10.1051/matecconf/2017/13400036

[13] Zaranka J, Peceliunas R, Matijosius J. Analysis of the influence of fatigue on passenger transport drivers' performance capacity. Transport. 2012;27(4):351-6. DOI: 10.3846/16484142. 2012.750625

[14] Matijosius J, Ciziuniene K, Petraska A. The comparison of the accident rates in heavy transport sectors of Europe and USA. 3rd
International Conference on Traffic and Transport Engineering (ICTTE) Belgrade, Serbia, NOV 24-25, 2016, Proceedings of the Third International Conference on Traffic and Transport Engineering (ICTTE). 2016;1097-101.

[15] Brumercikova E, Bukova B, Kondek P, Droździel P. Application of NFC technology in railway passenger transport by introducing new products. Communications - Scientific Letters of the University of Zilina. 2017;19(2):32-35.

[16] Filipowicz B. Modele stochastyczne w badaniach operacyjnych. Warszawa: WNT, 1996. 328 p.

[17] Jia D, Ngoduy D, Vu HL. A multiclass microscopic model for heterogeneous platoon with vehicle-to-vehicle communication. Transportmetrica B-Transport Dynamics, 2019;7(1):448-72.

[18] https://www.orpa.pl/

[19] Kampf R, Hlatka M, Savin G. Proposal for optimizing specific distribution routes by means of the specific method of operational analysis. Communications - Scientific Letters of the University of Zilina. 2017;19(2):133-38.

[20] Chovancová M, Klapita V. Draft model for optimization of the intermodal transport chains by applying the network analysis. 20th International Scientific on Conference Transport Means 2016, Juodkrante, Lithuania; 5-7 October 2016:112-16.

[21] https://spidersweb.pl/

[22] Ulmer M, Streng S. Same-Day delivery with pickup stations and autonomous vehicles. Comput. Oper. Res. 2019;108:1-19.

[23] Chen H, Yan D. Interrelationships between influential factors and behavioral intention with regard to autonomous vehicles. Int. J. Sustain. Transport. 2019;13(7):511-27. DOI: https://doi.org/10. 1080/15568318.2018.1488021

[24] Liu Z, Miwa T, Zeng W, Bell MGH, Morikawa T. Dynamic shared autonomous taxi system considering on-time arrival reliability. Transp. Res. Part C: Emerg. Technol. 2019;103:281-97, DOI: https: //doi.org/10.1016/j.trc.2019.04.018

[25] Bsaybes S, Quilliot A, Wagler A. Fleet management for autonomous vehicles using flows in time-expanded networks. Top (Madr). 2019;27(2):288-311.

[26] Lau HYK, Wong VWK, Lee ISK. Immunity-based autonomous guided vehicles control. Appl. Soft Comput. 2007;7(1):41-57. DOI: https://doi.org/10.1016/j.asoc.2005.02.003

[27] Freschi F, Repetto M. Multi objective optimization by a modified artificial immune system algorithm. International Conference on Artificial Immune Systems ICARIS 2005: Artificial Immune Systems. 2005:248-61.

[28] Subramani D, Lermusiaux P. Risk-optimal path planning in stochastic dynamic environments. Comput. Methods Appl. Mech. Eng. 2019;353:391-415. DOI: 10.1016/j.cma.2019.04.033

[29] Qiu L, Hsu W, Huang S, Wang H. Scheduling and routing algorithms for AGVs: a survey. Int. J. Prod. Res. 2002;40(3):745-60. https://doi.org/10.1080/00207540110091712

[30] Malmborg CJ. A model for the design of zone control automated guided vehicle systems. Int. J. Prod. Res. 1990;28(10):1741-58. https://doi.org/10.1080/00207549008942830

[31] Zhang Y, Malikopoulos A, Cassandras Ch. Optimal control and coordination of connected and automated vehicles at urban traffic intersections. American Control Conference (ACC). 2016.

[32] Grunow M, Günther HO, Lehmann M. Strategies for dispatching AGVs at automated seaport container terminals. OR-Spectrum. 2006;28:587-610.

[33] Nieoczym A. Application of a transportation flux for determining qualitative indices. Komunikacie. 2005;7(1):47-8. 
[34] Stopka O, Stopkova M, Kampf R. Application of the operational research method to determine the optimum transport collection cycle of municipal waste in a predesignated urban area. Sustainability. 2019;11(8):2275. DOI: 10.3390/su11082275

[35] Dobrodolac M, Lazarevic D, Svadlenka L, Zivanovic M. A study on the competitive strategy of the universal postal service provider. Technol. Anal. Strateg Manage. 2016;28(8):935-49. DOI: 10.1080/09537325.2016.1180357

[36] Madlenáková L, Madleňák R, Rudawska A, Droździel P. On the problem of quantitative analysis of the concentration on the postal and logistics market. Transport Means - Proceedings of the International Conference. 2017:693-8.
[37] L'upták V, Droździel P, Stopka O, Stopková M, Rybicka I. Approach methodology for comprehensive assessing the public passenger transport timetable performances at a regional scale. Sustainability. 2019;11(13):3532. DOI: 10.3390/su11133532

[38] Rybicka I, Droździel P, Stopka O, Lupták V. Methodology to propose a regional transport organization within specific integrated transport system: A case study. Transp. Probl. 2018;13(4):115-25. DOI: $10.20858 /$ tp.2018.13.4.11

[39] Stopka 0. Approach technique of specifying a proper autonomous cart type for its service in the logistics center. The Archives of Automotive Engineering - Archiwum Motoryzacji. 2019;84(2):23-31. DOI: https://doi.org/10.14669/AM.VOL84. ART2 